\title{
BMJ
}

\section{Cost effectiveness analysis of including boys in a human papillomavirus vaccination programme in the United States}

\author{
Jane J Kim, assistant professor Sue J Goldie, professor
}

Harvard School of Public Health, Department of Health Policy and Management, Center for Health Decision Science, 718 Huntington Avenue, Boston, MA 02115, USA Correspondence to: J J Kim jkim@hsph.harvard.edu

Cite this as: BMJ 2009;339:b3884 doi:10.1136/bmj.b3884

\section{ABSTRACT}

Objective To assess the cost effectiveness of including preadolescent boys in a routine human papillomavirus (HPV) vaccination programme for preadolescent girls. Design Cost effectiveness analysis from the societal perspective.

Setting United States.

Population Girls and boys aged 12 years. Interventions HPV vaccination of girls alone and of girls and boys in the context of screening for cervical cancer. Main outcome measure Incremental cost effectiveness ratios, expressed as cost per quality adjusted life year (QALY) gained.

Results With $75 \%$ vaccination coverage and an assumption of complete, lifelong vaccine efficacy, routine $H P V$ vaccination of 12 year old girls was consistently less than $\$ 50000$ per QALY gained compared with screening alone. Including preadolescent boys in a routine vaccination programme for preadolescent girls resulted in higher costs and benefits and generally had cost effectiveness ratios that exceeded $\$ 100000$ per QALY across a range of HPV related outcomes, scenarios for cervical cancer screening, and assumptions of vaccine efficacy and duration. Vaccinating both girls and boys fell below a willingness to pay threshold of $\$ 100000$ per QALY only under scenarios of high, lifelong vaccine efficacy against all HPV related diseases (including other non-cervical cancers and genital warts), or scenarios of lower efficacy with lower coverage or lower vaccine costs. Conclusions Given currently available information, including boys in an HPV vaccination programme generally exceeds conventional thresholds of good value for money, even under favourable conditions of vaccine protection and health benefits. Uncertainty still exists in many areas that can either strengthen or attenuate our findings. As new information emerges, assumptions and analyses will need to be iteratively revised to continue to inform policies for HPV vaccination.

\section{INTRODUCTION}

Persistent infection with high risk oncogenic types of human papillomavirus (HPV) has been established as a necessary factor in causing cervical cancer. Two types, HPV 16 and HPV 18, are responsible for about $70 \%$ of the cases of cervical cancer worldwide and contribute to over $80 \%$ of anal cancers; $30 \%$ of vulvar, vaginal, and oropharyngeal cancers; and $20 \%$ of oral cancers. ${ }^{1-3}$ Furthermore, two low risk non-oncogenic types, HPV 6 and HPV 11, are associated with most cases of anogenital warts and juvenile onset recurrent respiratory papillomatosis, a rare yet severe respiratory condition. ${ }^{4}$

Vaccines that target HPV 16 and HPV 18 have shown high, sustained efficacy against persistent type specific infections and precancerous cervical, vulvar, and vaginal lesions among females without previous exposure to these HPV types. ${ }^{5-11}$ The quadrivalent vaccine also targets HPV 6 and HPV 11 and has high efficacy against incident genital warts among females. ${ }^{5}$ Because the vaccine is most efficacious before exposure to HPV, current guidelines prioritise girls aged 11 to 12 as the primary target group for HPV vaccination; previous cost effectiveness analyses have consistently reported that HPV vaccination of preadolescent girls provides good value for money. ${ }^{12-22}$ Opinions on the optimal age limit for a catch-up vaccination programme in girls are more varied, extending to age 18 or 26 in the United States. ${ }^{2324}$

Recent data on the use of the HPV vaccine in males suggest high efficacy against vaccine type infections and external genital lesions. ${ }^{2526}$ Despite limited data, the HPV vaccine is licensed and recommended for boys in several countries. In the US, the Food and Drug Administration has not yet approved the HPV vaccine for boys but is expected to consider it in the near future; agencies responsible for guidelines, such as the Advisory Committee on Immunization Practices and the American Cancer Society, will need to advise whether or not HPV vaccination should be recommended for boys. Because HPV is a sexually transmitted infection, vaccinating boys may lead not only to direct health benefits (for themselves) but also to indirect health benefits (for sexual partners) through reduced transmission of HPV. To assess the value of adding boys to an HPV vaccination programme, both the incremental health benefits that may accrue to men and women and the economic costs of the programme should be compared with those associated with vaccinating girls alone. In particular in the US, as discussions about health reform proceed, there has been a call for analyses to compare the value of different health interventions. ${ }^{2728}$ 
The most important health benefits from HPV vaccination of adolescents (that is, the prevention of cancer) will not be observed for years, possibly decades. Disease simulation models that are calibrated to fit empirical data can be used within a decision analytical framework to synthesise the best available data, compare the health and economic outcomes of using different interventions, and explore "what if" scenarios that would otherwise be infeasible or unethical to pursue in a clinical study. We adopted such a framework to assess the cost effectiveness of including preadolescent boys in an HPV vaccination programme for preadolescent girls in the US.

\section{METHODS}

We used a series of published disease simulation models that synthesise epidemiological, clinical, and economic data to evaluate the incremental benefits and cost effectiveness of including preadolescent (age 12 years) boys in a routine HPV vaccination programme for preadolescent girls in the context of screening for cervical cancer in the US. The primary analysis included outcomes related to cervical disease, as well as other cancers associated with HPV 16 and HPV 18 for both women and men, which are relevant to both bivalent and quadrivalent vaccines. Secondary analyses included HPV 6 and HPV 11 associated genital warts as well as HPV 6 and HPV 11 associated juvenile onset recurrent respiratory papillomatosis. We assessed the impact of uncertainties such as vaccine efficacy in boys, the long term impact on health outcomes not yet observed in clinical trials (anal, oral, and oropharyngeal cancers and juvenile onset recurrent respiratory papillomatosis), achievable vaccination coverage, duration of vaccine protection, and cross protective effects against high risk HPV infections and cervical disease other than those related to HPV 16 and HPV 18. In sensitivity analysis we assessed the influence of additional uncertain variables, such as costs associated with screening and vaccination.

As recommended for economic evaluations of public health interventions in the $\mathrm{US}^{29}$ we adopted a societal perspective, including costs regardless of the payor, and discounted costs and health benefits by $3 \%$ annually. We calculated incremental cost effectiveness ratios for each strategy as the additional cost divided by the additional health benefit associated with one strategy compared with the next less costly strategy. We eliminated those strategies that were more costly and less effective (that is, strongly dominated) or less costly and less cost effective (that is, weakly dominated) than an alternative strategy. Although there is no consensus on a cut-off point for good value for resources, we present our results in the context of commonly cited thresholds per quality adjusted life year (QALY) of \$ 50000 and $\$ 100000 .^{30}$

\section{Models}

We used a dynamic transmission model to simulate sexual transmission of HPV 16 and HPV 18 infections between women and men and an individual based microsimulation model to reflect HPV induced cervical disease in women, both of which were calibrated to fit to epidemiological data. We used incidence based models to estimate the health and economic burden of non-cervical diseases for both sexes. Details of the models have been published. ${ }^{1231-33}$

The dynamic model is an age structured, population based model that simulates multiple birth cohorts of females and males from birth until death. According to patterns of sexual behaviour in the US, ${ }^{34}$ females and males form heterosexual partnerships as they age, and HPV 16 or HPV 18 may be transmitted depending on the number of new contacts per year, prevalence of HPV 16 and HPV 18 in the opposite sex at any given time, and the probabilities of transmitting the two types to an uninfected partner. After a first infection with HPV and clearance, individuals develop partial type specific natural immunity, effectively reducing their susceptibility to future infection with the same types. Women with an HPV infection can develop cervical intraepithelial neoplasia grade I, or grade II or III, which may regress naturally, and those with cervical intraepithelial neoplasia II or III may develop invasive cancer. Death can occur in any year from disease specific or background mortality.

The individual based stochastic model has a similar structure but includes all HPV types (categorised as HPV 16, HPV 18, other high risk types, and low risk types), represents the incidence of HPV as a function of age and individual level characteristics, tracks each individual's history (for example, vaccination, screening, treatment, and past abnormalities), and accommodates complex secondary prevention strategies. ${ }^{123233}$ The dynamic model was used to estimate reductions in age specific incidence of HPV 16 and HPV 18 with vaccination strategies, reflecting direct benefits to those vaccinated as well as indirect benefits to those not vaccinated (herd immunity). The generated reductions in incidence of HPV for females under scenarios of vaccinating girls alone or with boys served as inputs to the stochastic model, which we then used to compare vaccination strategies in the context of screening for cervical cancer.

\section{Model inputs and assumptions}

Initial model variables were based on epidemiological studies, cancer registries, population surveys, and demographic statistics from the US, where possible. The models were calibrated using a likelihood based approach to fit to empirical data, such as age specific prevalence of HPV, age specific incidence of cervical cancer, and HPV type distribution observed in the US population. ${ }^{135-40}$ The parameterisation, calibration, and validation approaches have been described elsewhere. ${ }^{123132}$ (See web extra on bmj.com for details relevant to the current analysis.)

For non-cervical cancer conditions, data included incidence of other HPV associated female and male cancers, genital warts, and juvenile onset recurrent respiratory papillomatosis, the proportion of each disease attributable to HPV types targeted by the vaccine, 
disease specific quality of life, costs, and mortality (table 1). ${ }^{1-441-50}$ Costs (2006 prices) included direct medical costs associated with vaccination (for example, three doses at $\$ 120$ per dose, wastage, supplies, and administration $)^{51-54}$ and with screening, diagnosis, and treatment (for example, tests, procedures, admissions to hospital). Estimates of direct non-medical costs, such as patient time and transportation, were included for all interventions.

\section{Analysis}

To estimate the long term outcomes associated with vaccination and screening strategies we projected the lifetime health and economic consequences for all birth cohorts in the first 10 years of the vaccination programme, which was assumed to continue for 100 years. Total lifetime costs and health benefits associated with each vaccination strategy served as the basis for cost effectiveness calculations.

Strategies included HPV vaccination of 12 year old girls alone and with 12 year old boys. Our base case analysis was purposefully constructed to consider a best case scenario for routine vaccination; as such, we assumed a coverage rate of $75 \%$ for both sexes on the basis of achievable coverage in past successful vaccination programmes for adolescents ${ }^{556}$ but also explored the implications of lower coverage (50\%). We assumed vaccine efficacy against incidence of vaccine targeted HPV infections to be lifelong and 100\% among females and $85 \%$ among males without a history of those infections ${ }^{925}$; efficacy against disease outcomes associated with vaccine targeted HPV types was $100 \%$ for females and $90 \%$ for males. ${ }^{5-1026}$ In sensitivity analyses we relaxed favourable assumptions to provide key insights into the influence of uncertainties on policy results. In particular we explored an alternative scenario of $75 \%$ efficacy against HPV infection and disease outcomes in boys only and waning immunity at 20 years. We also explored the impact of vaccine crossprotective effects against cervical disease associated with high risk HPV types other than HPV 16 or HPV $18(12.1 \%$ to $54.0 \%){ }^{10}$

We evaluated the vaccination strategies in the context of routine screening for cervical cancer beginning at an average age of 20 and using cytology (HPV DNA testing for triage of equivocal results), with and without a switch to combined cytology and HPV DNA testing after age 30, based on US guidelines. ${ }^{345758}$ Abnormal cytology test results were managed according to recommended clinical guidelines (see web extra)..$^{59}$ Based on patterns of screening for cervical cancer reported for US women, ${ }^{60-62}$ we assumed that current screening involves $53 \%$ of women being screened annually, $17 \%$ every two years, $11 \%$ every three years, 14\% every five years, and 5\% never screened. Because future screening practice is likely to change in response to a decreased risk of cervical disease in the population after vaccination, we also considered HPV vaccination alongside scenarios of less frequent routine screening (every two or three years), starting at a later age (25), with combined cytology and HPV
DNA testing for primary screening in older women (after age 35). ${ }^{63}$

For both bivalent and quadrivalent vaccines we estimated QALYs gained and costs averted for HPV 16 and HPV 18 associated non-cervical cancers for both sexes using age specific incidence, ${ }^{41}$ taking into account cancer specific mortality and quality of life weightsthat is, utilities (table 1). ${ }^{1-441-50}$ To reflect the benefits of the quadrivalent vaccine on HPV 6 and HPV 11, we modelled the age specific incidence and duration of genital warts, ${ }^{43}$ including their impact on quality of life and costs, ${ }^{43457}$ and we estimated QALYs gained and costs averted with vaccination. Similarly, we estimated cases of juvenile onset recurrent respiratory papillomatosis averted per vaccinated female using data on the number of births per woman, ${ }^{64}$ annual incidence of juvenile onset recurrent respiratory papillomatosis per live child, ${ }^{46}$ costs per case, and effects on quality of life. ${ }^{479}$ Vaccination was assumed to fully reduce the number of cases that are attributable to vaccine targeted types; vaccine protection included both direct and indirect benefits estimated from the dynamic model. Because of the uncertainty related to the long term benefits of the vaccine on outcomes not yet observed in clinical trials (other non-cervical cancers and juvenile onset recurrent respiratory papillomatosis), we assessed the impact of more conservative assumptions, such as a $50 \%$ reduction in cases attributable to vaccine targeted types.

\section{RESULTS}

Under assumptions of 75\% vaccination coverage and complete, lifelong vaccine protection, routine HPV vaccination of 12 year old girls who are screened using cytology (HPV DNA testing for triage) at current rates in adulthood was associated with an incremental cost effectiveness ratio of $\$ 40310$ per QALY gained compared with screening alone when including only benefits related to cervical disease (table 2). Adding 12 year old boys to the vaccination programme provided benefits for higher costs and had a cost effectiveness ratio of $\$ 290290$ per QALY compared with vaccinating girls only.

When including other HPV 16 and HPV 18 related cancers among women, the cost per QALY of vaccinating girls alone decreased below $\$ 32000$ and of vaccinating both sexes remained above $\$ 200000$ under assumptions of 50\% efficacy against HPV 16 and HPV 18 non-cervical cancers (100\% efficacy against cervical disease) or 100\% efficacy against all cancers caused by HPV 16 and HPV 18 in women. When including cancer benefits for both sexes, the cost effectiveness ratio for vaccinating boys in addition to girls remained above $\$ 100000$ per QALY.

When current screening involved a switch to combined cytology and HPV DNA testing after age 30 (table 2), the cost effectiveness ratio for the strategy of vaccinating girls alone stayed consistently below $\$ 50000$ per QALY across all scenarios and was generally lower (more attractive) than the corresponding ratios in the previous analysis except when reflecting 
Table 1| Model variables for HPV (human papillomavirus) related health conditions in women and men*

\begin{tabular}{|c|c|c|}
\hline Health condition & Women & Men \\
\hline \multicolumn{3}{|l|}{ Cervical cancer: } \\
\hline Incidence rate (per 100000$) \dagger$ & $4.2-62.8$ & - \\
\hline 5 year survival $(\%)^{41} \ddagger$ & $16.5-92.0$ & - \\
\hline Quality of life adjustment $\mathrm{t}^{45}{ }^{48} \S$ & $0.48-0.76$ & - \\
\hline Cases attributable to HPV 16 and HPV $18(\%)^{1-3}$ & 70.0 & - \\
\hline Cost per case $(\$)^{42} \pi$ & $26540-45540$ & - \\
\hline \multicolumn{3}{|l|}{ Vulvar cancer: } \\
\hline Incidence rate (per 100000 ) age $\geq 20^{41}$ & $0.2-19.6$ & - \\
\hline 5 year survival $(\%)^{41}$ & 77.8 & - \\
\hline Quality of life adjustment ${ }^{48} \S$ & 0.68 & - \\
\hline Cases attributable to HPV 16 and HPV $18(\%)^{23}$ & 32.0 & - \\
\hline Cost per case $(\$)^{47} \mathbb{\|}$ & 20430 & - \\
\hline \multicolumn{3}{|l|}{ Vaginal cancer: } \\
\hline Incidence rate (per 100000 ) age $\geq 20^{41}$ & $0.1-6.0$ & - \\
\hline 5 year survival $(\%)^{41}$ & 55.7 & - \\
\hline Quality of life adjustment ${ }^{48} \S$ & 0.68 & - \\
\hline Cases attributable to HPV 16 and HPV $18(\%)^{23}$ & 32.0 & - \\
\hline Cost per case $(\$)^{47} \mathbb{\|}$ & 23440 & - \\
\hline \multicolumn{3}{|l|}{ Penile cancer: } \\
\hline Incidence rate (per 100000 ) age $\geq 20^{41}$ & - & $0.0-7.6$ \\
\hline 5 year survival $(\%)^{41}$ & - & 75.0 \\
\hline Quality of life adjustment ${ }^{48} \S$ & - & 0.68 \\
\hline Cases attributable to HPV 16 and HPV $18(\%)^{23}$ & - & 25.2 \\
\hline Cost per case $(\$)^{47} \mathbb{\pi}$ & - & 17110 \\
\hline \multicolumn{3}{|l|}{ Anal cancer: } \\
\hline Incidence rate (per 100000 ) age $\geq 20^{41}$ & $0.0-5.6$ & $0.1-4.3$ \\
\hline 5 year survival $(\%)^{41}$ & 66.2 & 64.1 \\
\hline Quality of life adjustment ${ }^{48} \S$ & & \\
\hline Cases attributable to HPV 16 and HPV $18(\%)^{23}$ & & \\
\hline Cost per case $(\$)^{47} \mathbb{\pi}$ & & \\
\hline \multicolumn{3}{|l|}{ Oral cancer: } \\
\hline Incidence rate (per 100000 ) age $\geq 20^{41}$ & $0.2-13.9$ & $0.1-17.7$ \\
\hline 5 year survival $(\%)^{41}$ & 62.6 & 57.6 \\
\hline Quality of life adjustment§ & & \\
\hline Cases attributable to HPV 16 and HPV $18(\%)^{3}$ & & \\
\hline Cost per case $(\$)^{47} \mathbb{T}$ & & \\
\hline \multicolumn{3}{|l|}{ Oropharyngeal cancer: } \\
\hline Incidence rate (per 100000 ) age $\geq 20^{41}$ & $0.0-1.1$ & $0.0-2.9$ \\
\hline 5 year survival $(\%)^{41}$ & 62.6 & 57.6 \\
\hline Quality of life adjustment§ & & \\
\hline Cases attributable to HPV 16 and HPV $18(\%)^{3}$ & & \\
\hline Cost per case $(\$)^{47} \mathbb{\pi}$ & & \\
\hline \multicolumn{3}{|l|}{ Genital warts: } \\
\hline Prevalence rate $(\text { per } 1000)^{43}$ & $0.07-6.20$ & 0.13-5.01 \\
\hline Quality of life adjustment ${ }^{4345} \S$ & & \\
\hline Cases attributable to HPV 6 and HPV $11(\%)^{444}$ & & \\
\hline Cost per case $(\$)^{47} \mathbb{T}$ & & \\
\hline \multicolumn{3}{|l|}{ Juvenile onset recurrent respiratory papillomatosis: } \\
\hline Incidence rate (per 100000 ) age $0-14^{46}$ & & \\
\hline Quality of life adjustment ${ }^{49} \S$ & & \\
\hline Cases attributable to HPV 6 and HPV $11(\%)^{4}$ & & \\
\hline Cost per case $(\$)^{47} \boldsymbol{q}$ & & \\
\hline
\end{tabular}

*Range represents age specific values; rates are annual unless stated otherwise. Some data are not applicable to both sexes.

tIncidence of cervical cancer represents model generated projections by calibrated stochastic model in absence of screening or vaccination (natural history). †Five year survival for cervical cancer varied according to stage $\left(92.0 \%\right.$ for local, $55.7 \%$ for regional, $16.5 \%$ for distant). ${ }^{41}$

$\S Q u a l i t y$ of life adjustment assumes health state utility weight of 0 (death) to 1 (perfect health). Health state utility weight for cervical cancer varied according to stage: 0.76 for local cancer and 0.67 for regional cancer for five years, ${ }^{45}$ and 0.48 for distant cancer over lifetime with disease. ${ }^{48}$ For non-cervical cancers, average health state utility weight of 0.68 over the lifetime with disease is assumed, to reflect a weighted average of stage specific utilities and stage distribution of disease. ${ }^{48}$ For genital warts, health state utility weight of 0.91 over three months is assumed ${ }^{43} 45$; for juvenile onset recurrent respiratory papillomatosis, health state utility weight of 0.69 over four years is assumed. ${ }^{49}$ Disease specific utility weights were multiplied to baseline age specific utility weights to estimate overall utility. ${ }^{50}$

TCost per case is expressed in dollars at 2006 prices and represents average discounted lifetime costs of a new case of disease, including direct medical costs such as cost of procedures, admissions to hospital, and visits to the doctor. Treatment costs of cervical cancer varied according to stage $(\$ 26540$ for local, $\$ 28430$ for regional, $\$ 45540$ for distant) and included direct non-medical costs, such as patient time and transportation. ${ }^{42}$ 
Table 2 |Cost effectiveness of including boys in a vaccination programme against human papillomavirus (HPV) types 16 and 18 in the context of current screening for cervical cancer*

\begin{tabular}{|c|c|c|c|c|c|}
\hline \multirow[b]{2}{*}{ Strategy† } & \multicolumn{3}{|c|}{ Cancers in women only } & \multicolumn{2}{|c|}{ Cancers in both sexes } \\
\hline & Cervicalł & $\begin{array}{c}\text { Including other HPV } 16 \\
\text { and HPV } 18 \text { cancers } \\
(50 \% \text { efficacy }) \S\end{array}$ & $\begin{array}{c}\text { Including other HPV } 16 \\
\text { and HPV } 18 \text { cancers } \\
(100 \% \text { efficacy }) \Uparrow\end{array}$ & $\begin{array}{c}\text { Including other HPV } 16 \\
\text { and HPV } 18 \text { cancers } \\
(50 \% \text { efficacy }) \S\end{array}$ & $\begin{array}{c}\text { Including other HPV } 16 \\
\text { and HPV } 18 \text { cancers } \\
(90-100 \% \text { efficacy)ף }\end{array}$ \\
\hline \multicolumn{6}{|l|}{$\begin{array}{l}\text { Current screening using cytology } \\
\text { with HPV DNA testing for triage: }\end{array}$} \\
\hline No vaccination+screening & - & - & - & - & - \\
\hline Vaccination of girls aged $12+$ screening & 40310 & 31530 & 25680 & 27370 & 20990 \\
\hline Vaccination of girls and boys aged $12+$ screening & 290290 & 242520 & 208110 & 164580 & 114510 \\
\hline
\end{tabular}

Current screening using cytology with HPV DNA

testing for triage until age 30 , then combined

cytology and HPV DNA testing after age 30:

\begin{tabular}{|c|c|c|c|c|c|}
\hline No vaccination+screening & - & - & - & - & - \\
\hline Vaccination of girls aged $12+$ screening & 42450 & 30370 & 23310 & 25270 & 18130 \\
\hline Vaccination of girls and boys aged $12+$ screening & 350040 & 281170 & 234760 & 179510 & 120300 \\
\hline
\end{tabular}

*Values represent incremental cost effectiveness ratios (additional cost divided by additional health benefit compared with next less costly strategy) expressed as cost (\$) per quality adjusted life year (QALY). Costs expressed in 2006 dollars.

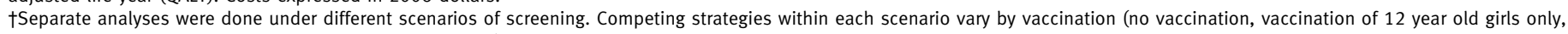

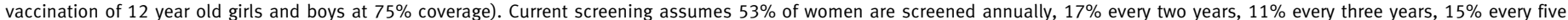
years, and $5 \%$ are never screened.

łIncludes outcomes related to cervical disease only and assumes $100 \%$ lifelong vaccine efficacy against HPV 16 and HPV 18 related cervical disease.

§Includes outcomes related to cervical disease and other HPV 16 and HPV 18 related cancers (vulvar and vaginal cancers for women; penile cancer for men; and anal, oral, and

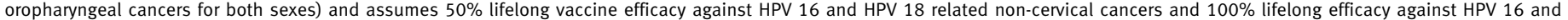
HPV 18 related cervical disease.

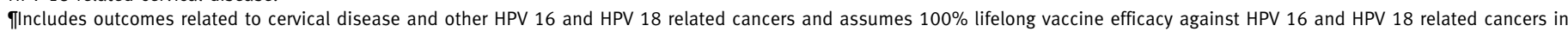
women and $90 \%$ lifelong vaccine efficacy against HPV 16 and HPV 18 related cancers in men.

cervical outcomes only. In contrast, the strategy of vaccinating both sexes had a higher (less attractive) ratio, ranging from \$120000 per QALY (when including all HPV 16 and HPV 18 related cancers at full efficacy) to $\$ 350000$ per QALY (when including only cervical outcomes).

Impact of decreased vaccine efficacy in boys

To reflect uncertainty about vaccine efficacy in males the analyses were repeated in a scenario of vaccine efficacy lowered to $75 \%$ in boys. Figure 1 indicates the cost

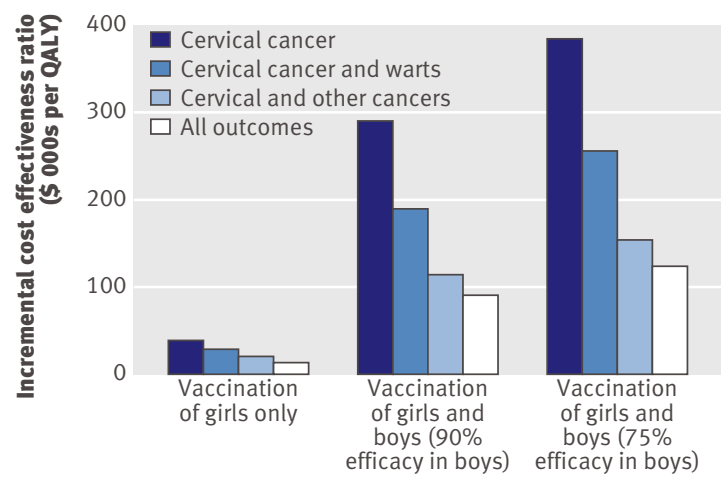

Impact of decreased HPV vaccine efficacy in boys on cost effectiveness ratios. Height of bars indicates cost effectiveness ratios associated with strategies of vaccinating girls alone and with boys in the context of current cytology screening (HPV DNA testing for triage), under two levels of vaccine efficacy in boys: $90 \%$ against vaccine type disease outcomes ( $85 \%$ against vaccine type HPV infections), and $75 \%$ against vaccine type infections and disease outcomes. In both scenarios, vaccine efficacy of $100 \%$ against vaccine type infections and disease outcomes in girls was assumed effectiveness ratios associated with the strategies of vaccinating girls alone and vaccinating both sexes in the context of current screening with cytology (HPV DNA testing for triage), when assuming 90\% and $75 \%$ vaccine efficacy in boys. For this analysis, HPV 6 and HPV 11 associated genital warts and all health outcomes combined are also reported, including HPV 6 and HPV 11 associated juvenile onset recurrent respiratory papillomatosis. Lowering the vaccine efficacy in males from $90 \%$ to $75 \%$ resulted in higher (less attractive) cost effectiveness ratios associated with inclusion of boys in the vaccination programme. For example, when reflecting benefits associated with cervical disease only, vaccinating both sexes (compared with vaccinating girls alone) increased from $\$ 290290$ to $\$ 382860$ per QALY as vaccine efficacy decreased from $90 \%$ to $75 \%$ in boys. When reflecting all health outcomes in women and men, the ratio for a strategy of including boys was $\$ 90870$ per QALY assuming 90\% vaccine efficacy against all HPV 16, HPV 18, HPV 6, and HPV 11 related diseases in males; this ratio increased to $\$ 123940$ per QALY when vaccine efficacy was decreased to $75 \%$.

Impact of vaccination coverage, duration of protection, and other uncertainties

When coverage for routine vaccination was assumed to reach only $50 \%$ in both sexes, the cost of vaccinating boys remained more than $\$ 220000$ per QALY when only cervical outcomes were included; this ratio decreased to $\$ 62070$ per QALY when efficacy against all outcomes for both sexes was high and lifelong but exceeded $\$ 92000$ when efficacy against warts in males and all non-cervical cancers was 50\%. Duration of 
Table $3 \mid$ Cost effectiveness of human papillomavirus (HPV) vaccination and revised screening strategies*

\begin{tabular}{|c|c|c|c|c|}
\hline \multirow[b]{2}{*}{ Strategy $†$} & \multirow[b]{2}{*}{$\begin{array}{c}\text { Cervicalł } \\
\text { (100\% efficacy) }\end{array}$} & \multicolumn{3}{|c|}{ All HPV related conditions in women and men§ } \\
\hline & & $50 \%$ efficacy & $\begin{array}{c}75 \% \text { efficacy** } \\
\text { (boys only) }\end{array}$ & $\begin{array}{c}90 \% \text { efficacyt† } \\
\text { (boys only) }\end{array}$ \\
\hline No vaccination+screening every three years & - & - & - & - \\
\hline Vaccination of girls aged $12+$ screening every three years & 37940 & 17560 & 11930 & 11930 \\
\hline No vaccination+screening every two years & Dominated & Dominated & Dominated & Dominated \\
\hline Vaccination of girls and boys aged $12+$ screening every three years & Not cost effective & 131010 & 122680 & 88930 \\
\hline Vaccination of girls aged $12+$ screening every two years & 190780 & Dominated & Dominated & Dominated \\
\hline Vaccination of girls and boys aged $12+$ screening every two years & 390440 & 220940 & 212910 & 220940 \\
\hline
\end{tabular}

*Values represent incremental cost effectiveness ratios (additional cost divided by additional health benefit compared with next less costly strategy) expressed as cost (\$) per quality

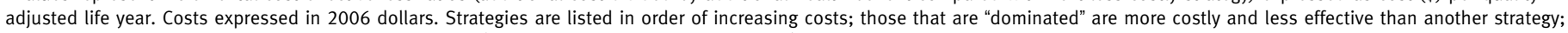
those that are "not cost effective" are less cost effective (have higher cost effectiveness ratios) than a more costly strategy.

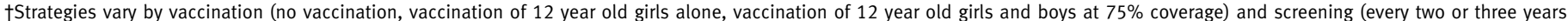
using cytology with HPV DNA testing for triage starting at age 25, with a switch to combined cytology and HPV DNA testing at age 35).

flncludes outcomes related to cervical disease only and assumes 100\% lifelong vaccine efficacy against HPV 16 and HPV 18 related cervical disease.

§Includes outcomes related to cervical disease and other HPV 16 and HPV 18 related cancers, and HPV 6 and HPV 11 related genital warts and juvenile onset recurrent respiratory papillomatosis among women and men.

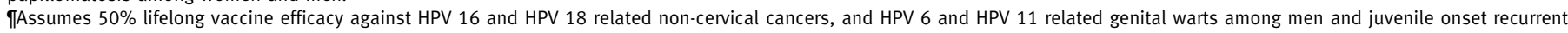
respiratory papillomatosis, and 100\% lifelong vaccine efficacy against HPV 16 and HPV 18 related cervical disease and HPV 6 and HPV 11 related genital warts among women.

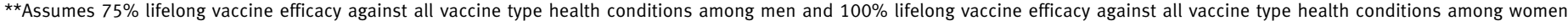

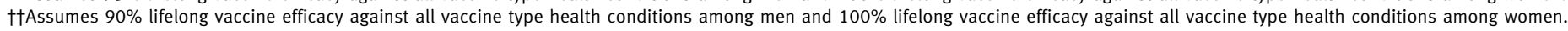

vaccine protection had a considerable impact on vaccination strategies, resulting in high cost effectiveness ratios even when including all HPV related outcomes. When vaccine induced immunity waned completely after 20 years, the cost effectiveness ratio of vaccinating girls alone was over $\$ 120000$ per QALY compared with current screening practice, and vaccinating both sexes cost over $\$ 350000$ per QALY.

When the cost per vaccinated individual was lowered to $\$ 360$ (including three doses, supplies, administration, patient time, and transportation; implying a cost per dose of roughly $\$ 87$ ), the cost of vaccinating both sexes decreased to $\$ 63000$ per QALY under an assumption of high efficacy against all outcomes; this improvement was attenuated when efficacy against warts in males and all non-cervical cancers was assumed to be $50 \%$, although the ratio remained below $\$ 100000$ per QALY. When the cost per vaccinated individual was increased to $\$ 600$, the cost of vaccinating both sexes exceeded $\$ 110000$ per QALY, even under favourable assumptions of high, lifelong vaccine efficacy against all conditions. Potential cross protective effects of the vaccine on infections and cervical disease related to oncogenic HPV types other than HPV 16 and HPV 18 had only a modest impact on the cost effectiveness ratios, and even using our upper bound estimate for cross protection, vaccination of both sexes remained well over $\$ 250000$ per QALY when only cervical outcomes were included. Varying costs associated with screening and treatment for cervical cancer also had minimal impact on the overall results.

\section{Cost effectiveness of HPV vaccination and screening strategies}

To reflect newly proposed algorithms for improving secondary prevention of cervical cancer, an analysis was carried out to evaluate different combinations of vaccination and screening strategies. For screening we included cytology (HPV DNA testing for triage) every two or three years starting at age 25 , with a switch to combined cytology and HPV DNA testing at age 35 (table 3). In addition to our primary outcome of cervical disease, we reported the benefits for all possible health conditions associated with HPV 6, HPV 11, HPV 16, and HPV 18 for women and men under varying assumptions of vaccine efficacy.

Under base case assumptions of 75\% vaccination coverage and complete, lifelong vaccine efficacy against cervical disease only, a strategy of vaccinating 12 year old girls with screening every three years in adulthood would cost \$37940 per QALY compared with screening every three years without vaccination, and would be less costly and more effective than a strategy of screening every two years without vaccination. Vaccinating both sexes, as well as vaccinating girls alone in the context of more frequent screening, were either not cost effective or exceeded \$190 000 per QALY.

When other HPV related outcomes were taken into consideration, inclusion of boys in the vaccination programme in the context of screening every three years was less costly and more effective than vaccinating girls alone with screening every two years, but cost over $\$ 120000$ per QALY when vaccine efficacy was 50\% against health outcomes associated with HPV 6, HPV 11, HPV 16, and HPV 18 (except for cervical disease and warts in females) or was $75 \%$ in males only. When we assumed lifelong vaccine efficacy of $100 \%$ for females and $90 \%$ for males (best case scenario), vaccinating both sexes along with screening every three years resulted in a decrease in cost per QALY to $\$ 88930$. The cost per QALY of vaccinating both sexes along with screening every two years remained more than $\$ 210000$ over all assumptions of vaccine efficacy against other HPV related conditions.

\section{DISCUSSION}

Our results suggest that if vaccine coverage and efficacy are high among preadolescent girls (12 years), 
then including boys in an HPV vaccination programme is unlikely to provide good value for resources compared with vaccinating girls only. This finding was stable even when we included potential vaccine benefits against other HPV related conditions among both women and men and under different scenarios of screening for cervical cancer. When we assumed lower vaccine efficacy, waning immunity, or higher vaccine costs, vaccination of boys consistently exceeded \$250 000 per QALY when reflecting benefits to cervical disease only and $\$ 100000$ per QALY when including other HPV related conditions. We found that vaccinating both sexes fell below a willingness to pay threshold of $\$ 100000$ per QALY only under scenarios of high, lifelong vaccine efficacy against all HPV related conditions (including other non-cervical cancers, genital warts, and juvenile onset recurrent respiratory papillomatosis), or scenarios of lower vaccine efficacy with lower coverage or lower vaccine costs.

Our finding that the cost effectiveness ratio of vaccinating preadolescent girls alone was below $\$ 50000$ per QALY when vaccine efficacy was high and long lasting is similar to the results of previous analyses. ${ }^{13-22}$ Of four studies that evaluated the cost effectiveness of including boys in an HPV vaccination programme in similar settings, three drew consistent conclusions. Under comparable base case assumptions, two of the studies ${ }^{1521}$ and our analysis found that vaccinating boys in addition to girls was unlikely to be cost effective compared with vaccinating girls alone. In contrast, another study ${ }^{18}$ reported that vaccination of girls and boys along with a temporary catch-up programme for both sexes to age 24 was less than $\$ 50000$ per QALY. Several important distinctions may contribute to the disparate findings, including (but not limited to) the following. Firstly, we incorporated recent data on efficacy against vaccine type HPV infection and diseases in males ${ }^{2526}$ that were lower than assumed in previous analyses, contributing to the less attractive cost effectiveness ratio for vaccinating both sexes compared with the earlier study. ${ }^{18}$ Likewise, differences in assumptions of screening test characteristics could influence the relative performance of vaccination strategies; the analysis in the earlier study ${ }^{18}$ assumed lower cytology sensitivity for detecting cervical intraepithelial neoplasia, which favours the vaccination strategies. Unlike the earlier study ${ }^{18}$ we did not include diminished quality of life among women with a diagnosis of cervical intraepithelial neoplasia; because vaccination will effectively reduce the number of cases (and therefore avert quality of life decrements of cervical intraepithelial neoplasia diagnosed through screening) exclusion of this assumption makes the vaccination strategies look less favourable. Other distinctions across analyses, including model structures and simulation techniques, assumptions of natural immunity, vaccination coverage and costs, analytical time horizon, and discounting rates, have been discussed in several review papers. ${ }^{65-69}$
When different vaccination and screening strategies were considered simultaneously, vaccinating preadolescent girls only along with cytology (HPV DNA testing for triage) every three years starting at age 25 and a switch to combined cytology and HPV DNA testing at age 35 consistently had a cost effectiveness ratio below $\$ 50000$ per QALY. This finding, corroborated by several studies, ${ }^{131617}$ shows the importance of re-evaluating new screening tests as they become available in the context of refined algorithms for screening frequency, starting age, and follow-up procedures in women after vaccination. Moreover, changes in the distribution of HPV genotypes after widespread vaccination may have important implications for the performance of screening tests, including the decreased positive predictive value of cytology testing. ${ }^{63}$ Alternative screening strategies involving HPV DNA testing alone as a primary screening test in older women ( $>35$ years) with cytology as a triage for abnormal test results, look promising on the basis of preliminary clinical data as well as modelling results ${ }^{17}$ and should be explored more thoroughly.

\section{Limitations of the study}

Limitations of our analysis that are related to uncertainties of the clinical course of included health conditions and vaccine properties in the long term have been discussed. ${ }^{12}$ Firstly, assumptions about sexual behaviour were simplified on the basis of large population surveys; however, variables of the dynamic model were calibrated to fit age specific prevalence of HPV in the population, such that the implications of our assumptions on sexual mixing were consistent with empirical data. Secondly, individuals in the population are likely to benefit differentially from the vaccine. A limitation of our analysis is that we only represented heterosexual partnerships and therefore did not reflect HPV transmission among men who have sex with men, who face a high risk of anal cancer and may realise a greater benefit from HPV vaccination. Such an analysis would require a more comprehensive model that includes a fuller range of sexual behaviours, which we acknowledge as an important priority for future work. Thirdly, data on incidence, mortality, and quality of life associated with HPV related cancers other than cervical cancer are also limited. We used multiple models to leverage the different levels of data available for each health condition, yet inconsistencies between model types and complexities associated with model linkage should be further explored. Fourthly, data on vaccine efficacy are reported primarily for females and include outcomes related only to HPV infections, precancerous lesions, and genital warts. Longer term data on vaccine properties of both the bivalent and quadrivalent vaccines will be important to incorporate when available, to provide more accurate estimates of their expected benefits and costs.

We purposefully bound the scope of this analysis to an assessment of the cost effectiveness of routine vaccination in preadolescent girls and boys at an optimistic coverage rate of $75 \%$, on the basis of achievable 


\section{WHAT IS ALREADY KNOWN ON THIS TOPIC}

Infection with high risk types of human papillomavirus (HPV) is associated with most cervical and anal cancers and a smaller fraction of other anogenital, oral, and oropharyngeal cancers

Vaccines that target HPV 16 and HPV 18 have shown high, sustained efficacy against persistent type specific infection and precancerous cervical, vulvar, and vaginal lesions among females

Current US guidelines for routine HPV vaccination prioritise girls aged 11 to 12 as the primary target group and will probably consider including boys in the near future

\section{WHAT THIS STUDY ADDS}

Under conditions of high vaccine coverage and efficacy in girls, including boys in an HPV vaccination programme generally exceeds conventional thresholds of good value for money

coverage of other successful vaccines among adolescents. ${ }^{556}$ Acknowledging the uncertainty in the ultimate achievable coverage, we included an analysis of $50 \%$ coverage and found that the cost effectiveness ratios for both vaccination strategies became more attractive because of the increased opportunities for benefits from herd immunity. The cost effectiveness of adding boys to the vaccination programme, however, decreased below $\$ 75000$ per QALY only if we assumed that vaccine efficacy against other HPV related outcomes for both females and males was high and lifelong; it exceeded $\$ 90000$ per QALY under assumptions of lower efficacy and remained above $\$ 200000$ per QALY when only the primary outcome of cervical cancer was reflected. We also did not consider temporary catch-up programmes, as we thought that data were inadequate for important assumptions that would be required, including rates for catch-up coverage stratified by age and sexual history. Nonetheless, because of the higher coverage of girls in a catch-up campaign and consequent diminished opportunity for herd immunity benefits from vaccinating boys, we expect that vaccinating boys would be even less attractive under catch-up scenarios.

Although we did not include decrements in quality of life associated with diagnosed precancerous cervical lesions, which would favour the vaccination strategies, we also did not incorporate diminished quality of life due to potential adverse events associated with vaccination, which would favour the screening strategies. To date causal links between the vaccine and serious side effects have been inconclusive, although a recent analysis using data from the US vaccine adverse event reporting system suggested higher rates of venous thromboembolic events and syncope among those who received the HPV vaccine. ${ }^{5170}$ Even minor side effects cause undue health and economic burden and may, in aggregate, outweigh the benefits of HPV vaccination since the population risk of cervical cancerand other HPV related conditions - in the United States is low. ${ }^{71}$ Inclusion of adverse events, and the cost of surveillance or reporting of adverse events, although likely to strengthen our results, is an important priority to revisit as data become available.
Our analysis does not address decision making at the individual level; indeed, families considering HPV vaccination for boys, and who are willing to pay for the vaccine, may consider the benefits of the vaccine worthwhile in terms of reducing the future risk of genital warts and possibly other health conditions. Instead, we emphasise the public health perspective of this analysis, which has the objective of informing general policy recommendations at the population level, enabling us to compare its value with other public health interventions vying for similar resources. Such an analysis is particularly relevant in the light of the recent prioritisation of "comparative effectiveness" research under the American Recovery and Reinvestment Act of 2009. ${ }^{72}$

\section{Conclusions}

Given the information currently available, our analysis favours HPV vaccination of preadolescent girls (with continued screening in adulthood) as a valuable intervention for its cost, consistent with findings from other cost effectiveness studies. ${ }^{13-22}$ Including boys in the vaccination programme, however, generally exceeded conventional thresholds of good value for money, even under favourable conditions of vaccine protection and health benefits. Uncertainty still exists in many areas that can either strengthen or attenuate our findings. As new data become available and new information emerges, assumptions and analyses will need to be iteratively revised to continue to inform policies on HPV vaccination.

Contributors: JJK and SJG conceived and designed the study; analysed and interpreted the data; drafted and critically revised the manuscript for important intellectual content; and approved the version to be published. Both authors had full access to all the data and take responsibility for the integrity of the data and the accuracy of the data analysis. JJK is the guarantor

Funding: The authors are supported by grants from the National Cancer Institute (R01CA93435), the Centers for Disease Control and Prevention, and the American Cancer Society, and by the Bill and Melinda Gates Foundation (30505) for related work in developing countries. The authors' work was independent of the funders, and the funding sources had no involvement in the study design; collection, analysis, or interpretation of data; writing of the report; or the decision to submit the paper for publication.

Competing interests: None declared.

Ethical approval: Not required.

Data sharing: No additional data available.

1 Clifford GM, Smith JS, Plummer M, Munoz N, Franceschi S. Human papillomavirus types in invasive cervical cancer worldwide: a metaanalysis. BrJ Cancer 2003;88:63-73.

2 Parkin DM, Bray F. Chapter 2: the burden of HPV-related cancers. Vaccine 2006;24(suppl 3):S11-25.

3 Watson M, Saraiya M, Ahmed F, Cardinez CJ, Reichman ME, Weir HK, et al. Using population-based cancer registry data to assess the burden of human papillomavirus-associated cancers in the United States: overview of methods. Cancer 2008;113(suppl 10):2841-54.

4 Lacey C), Lowndes CM, Shah KV. Chapter 4: burden and managemen of non-cancerous HPV-related conditions: HPV-6/11 disease. Vaccine 2006;24(suppl 3):S35-41.

5 Garland SM, Hernandez-Avila M, Wheeler CM, Perez G, Harper DM, Leodolter S, et al. Quadrivalent vaccine against human papillomavirus to prevent anogenital diseases. $N$ Engl J Med 2007;356:1928-43.

6 Future II Study Group. Quadrivalent vaccine against human papillomavirus to prevent high-grade cervical lesions. N Engl J Med 2007;356:1915-27.

7 Ault KA. Effect of prophylactic human papillomavirus L1 virus-likeparticle vaccine on risk of cervical intraepithelial neoplasia grade 2 , grade 3, and adenocarcinoma in situ: a combined analysis of four randomised clinical trials. Lancet 2007;369:1861-8. 
8 Paavonen J, Jenkins D, Bosch FX, Naud P, Salmeron J, Wheeler CM, et al. Efficacy of a prophylactic adjuvanted bivalent L1 virus-likeparticle vaccine against infection with human papillomavirus types 16 and 18 in young women: an interim analysis of a phase III doubleblind, randomised controlled trial. Lancet 2007;369:2161-70.

9 Harper DM, Franco EL, Wheeler CM, Moscicki AB, Romanowski B, Roteli-Martins CM, et al. Sustained efficacy up to 4.5 years of a bivalent $\mathrm{L} 1$ virus-like particle vaccine against human papillomavirus types 16 and 18: follow-up from a randomised control trial. Lancet 2006;367:1247-55.

10 Paavonen J, Naud P, Salmeron J, Wheeler CM, Chow SN, Apter D, et al. Efficacy of human papillomavirus (HPV)-16/18 AS04-adjuvanted vaccine against cervical infection and precancer caused by oncogenic HPV types (PATRICIA): final analysis of a double-blind, randomised study in young women. Lancet 2009;374:301-14.

11 Rowhani-Rahbar A, Mao C, Hughes JP, Alvarez FB, Bryan JT, Hawes SE, et al. Longer term efficacy of a prophylactic monovalent human papillomavirus type 16 vaccine. Vaccine 2009;27:5612-9.

12 Kim JJ, Goldie SJ. Health and economic implications of HPV vaccination in the United States. N Engl J Med 2008;359:821-32.

13 Goldie S), Kohli M, Grima D, Weinstein MC, Wright TC, Bosch FX, et al. Projected clinical benefits and cost-effectiveness of a human papillomavirus 16/18 vaccine. J Natl Cancer Inst 2004;96:604-15.

14 Sanders GD, Taira AV. Cost-effectiveness of a potential vaccine for human papillomavirus. Emerg Infect Dis 2003;9:37-48.

15 Taira AV, Neukermans CP, Sanders GD. Evaluating human papillomavirus vaccination programs. Emerg Infect Dis 2004;10:1915-23.

16 Kulasingam SL, Myers ER. Potential health and economic impact of adding a human papillomavirus vaccine to screening programs. JAMA 2003;290:781-9.

17 Goldhaber-Fiebert JD, Stout NK, Salomon JA, Kuntz KM, Goldie SJ. Cost-effectiveness of cervical cancer screening with human papillomavirus (HPV) DNA testing and HPV-16,18 vaccination. I Natl Cancer Inst 2008;100:308-20.

18 Elbasha EH, Dasbach EJ, Insinga RP. Model for assessing human papillomavirus vaccination strategies. Emerg Infect Dis 2007;13:28-41.

19 Brisson M, Van de Velde N, De Wals P, Boily MC. The potential cost effectiveness of prophylactic human papillomavirus vaccines in Canada. Vaccine 2007;25:5399-408.

20 Boot HJ, Wallenburg I, de Melker HE, Mangen MJ, Gerritsen AA, van der Maas NA, et al. Assessing the introduction of universal human papillomavirus vaccination for preadolescent girls in The Netherlands. Vaccine 2007;25:6245-56

21 Jit M, Choi YH, Edmunds WJ. Economic evaluation of human papillomavirus vaccination in the United Kingdom. BMJ 2008;337:a769.

22 Mennini FS, Giorgi Rossi P, Palazzo F, Largeron N. Health and economic impact associated with a quadrivalent HPV vaccine in Italy. Gynecol Oncol 2009;112:370-6.

23 Saslow D, Castle PE, Cox JT, Davey DD, Einstein MH, Ferris DG, et al. American Cancer Society guideline for human papillomavirus (HPV) vaccine use to prevent cervical cancer and its precursors. CA Cancer Clin 2007;57:7-28

24 Markowitz LE, Dunne EF, Saraiya M, Lawson HW, Chesson H, Unger ER. Quadrivalent human papillomavirus vaccine: recommendations of the Advisory Committee on Immunization Practices (ACIP). MMWR Recomm Rep 2007;56(RR-2):1-24.

25 Palefsky J, Giuliano AR. Efficacy of the quadrivalent HPV vaccine against HPV 6/11/16/18-related genital infection in young men. EUROGIN. Nice, France, 12-15 Nov, 2008. www.eurogin.com/2008/ EUROGIN2008_LastMinuteAbstracts.pdf. 2009.

26 Giuliano AR, Palefsky). The efficacy of quadrivalent HPV (types 6/11/ $16 / 18$ ) vaccine in reducing the incidence of HPV-related genital disease in young men. EUROGIN. Nice, France, 12-15 Nov, 2008. www.eurogin.com/2008/EUROGIN2008_LastMinuteAbstracts.pdf. 2009.

27 Mitka M. Comparative effectiveness. JAMA 2008;300:1290.

28 Barnett D, Chalkidou K, Rawlins M. Comparative effectiveness research: a useful tool. Health Aff 2009;28:600-1.

29 Gold MR, Siegel JE, Russel LB, Weinstein MC, eds. Cost-effectiveness in health and medicine. New York: Oxford University Press, 1996.

30 Eichler HG, Kong SX, Gerth WC, Mavros P, Jonsson B. Use of costeffectiveness analysis in health-care resource allocation decisionmaking: how are cost-effectiveness thresholds expected to emerge? Value Health 2004;7:518-28.

31 Kim JJ, Andres-Beck B, Goldie SJ. The value of including boys in an HPV vaccination programme: a cost-effectiveness analysis in a lowresource setting. Br J Cancer 2007;97:1322-8.

32 Kim JJ, Kuntz KM, Stout NK, Mahmud S, Villa LL, Franco EL, et al. Multiparameter calibration of a natural history model of cervical cancer. Am J Epidemiol 2007;166:137-50.
33 Goldie SJ, Kim JJ, Kobus K, Goldhaber-Fiebert JD, Salomon J, O’Shea MK, et al. Cost-effectiveness of HPV 16, 18 vaccination in Brazil. Vaccine 2007;25:6257-70.

34 Mosher WD, Chandra A, Jones J. Sexual behavior and selected health measures: men and women 15-44 years of age, United States, 2002. Adv Data 2005:1-55.

35 Dunne EF, Unger ER, Sternberg M, McQuillan G, Swan DC, Patel SS, et al. Prevalence of HPV infection among females in the United States. JAMA 2007;297:813-9.

36 Clifford G, Franceschi S, Diaz M, Munoz N, Villa LL. Chapter 3: HPV type-distribution in women with and without cervical neoplastic diseases. Vaccine 2006;24(suppl 3):S26-34.

37 Clifford GM, Gallus S, Herrero R, Munoz N, Snijders PJ, Vaccarella S, et al. Worldwide distribution of human papillomavirus types in cytologically normal women in the International Agency for Research on Cancer HPV prevalence surveys: a pooled analysis. Lancet 2005;366:991-8.

38 Clifford GM, Smith JS, Aguado T, Franceschi S. Comparison of HPV type distribution in high-grade cervical lesions and cervical cancer: a meta-analysis. BrJ Cancer 2003;89:101-5.

39 International Union against Cancer. Cancer incidence in five continents, vol. 1. New York: Springer-Verlag, 1966

40 Franceschi S, Castellsague X, DalMaso L, Smith JS, Plummer M, Ngelangel $C$, et al. Prevalence and determinants of human papillomavirus genital infection in men. Br/Cancer 2002;86:705-11.

41 National Cancer Institute. Surveillance, Epidemiology, End Results (SEER) cancer statistics review, 1975-2001. http://seer.cancer.gov/ csr/1975 2001/. 2005.

42 Goldie SJ, Kim JJ, Wright TC. Cost-effectiveness of human papillomavirus DNA testing for cervical cancer screening in women aged 30 years or more. Obstet Gynecol 2004;103:619-31.

43 Insinga RP, Dasbach EJ, Myers ER. The health and economic burden of genital warts in a set of private health plans in the United States. Clin Infect Dis 2003;36:1397-403.

44 Schiffman M, Castle PE, Jeronimo J, Rodriguez AC, Wacholder S. Human papillomavirus and cervical cancer. Lancet 2007;370:890-907

45 Myers ER, Green S, Lipkus I. Patient preferences for health states related to HPV infection: visual analogue scales versus time trade-off elicitation [abstract]. Proceedings of the 21st international papillomavirus conference. Mexico City, Mexico, 2004.

46 Derkay CS. Task force on recurrent respiratory papillomas. A preliminary report. Arch Otolaryngol Head Neck Surg 1995;121:1386-91.

$47 \mathrm{Hu}$ D, Goldie SJ. The economic burden of noncervical human papillomavirus disease in the United States. Am J Obstet Gynecol 2008;198:500.e1-7.

48 Gold MR, Franks P, McCoy Kl, Fryback DG. Toward consistency in costutility analyses: using national measures to create condition-specific values. Med Care 1998;36:778-92.

49 Bishai D, Kashima H, Shah K. The cost of juvenile-onset recurrent respiratory papillomatosis. Arch Otolaryngol Head Neck Surg 2000;126:935-9.

50 Fryback DG, Dasbach EJ, Klein R, Klein BE, Dorn N, Peterson K, et al. The Beaver Dam Health Outcomes Study: initial catalog of healthstate quality factors. Med Decis Making 1993;13:89-102.

51 US Centers for Disease Control and Prevention. HPV vaccine information for young women. 2009. www.cdc.gov/std/hpv/ STDFact-HPV-vaccine-young-women.htm

52 Wallace LA, Young D, Brown A, Cameron JC, Ahmed S, Duff R, et al. Costs of running a universal adolescent hepatitis $B$ vaccination programme. Vaccine 2005;23:5624-31.

53 Iskedjian M, Walker JH, Hemels ME. Economic evaluation of an extended acellular pertussis vaccine programme for adolescents in Ontario, Canada. Vaccine 2004;22:4215-27.

54 US Centers for Disease Control and Prevention. HIV counseling and testing system, version 4.0. Atlanta: National Center for HIV, STD, and TB Prevention, 1999.

55 US Centers for Disease Control and Prevention. National vaccination coverage among adolescents aged 13-17 years-United States, 2006. MMWR 2007;56:885-8.

56 US Centers for Disease Control and Prevention. Vaccination coverage among adolescents aged 13-17 years—United States, 2007. MMWR 2008;57:1100-3.

57 Saslow D, Runowicz CD, Solomon D, Moscicki AB, Smith RA, Eyre HJ, et al. American Cancer Society guideline for the early detection of cervical neoplasia and cancer. CA Cancer J Clin 2002;52:342-62.

58 American College of Obstetricians and Gynecologists. ACOG practice bulletin. Cervical cytology screening. No 45, Aug 2003. Int / Gynaecol Obstet 2003;83:237-47

59 Wright TC Jr, Massad LS, Dunton CJ, Spitzer M, Wilkinson EJ, Solomon D. 2006 consensus guidelines for the management of women with abnormal cervical cancer screening tests. Am J Obstet Gynecol 2007;197:346-55. 
60 Eltoum IA, Roberson J. Impact of HPV testing, HPV vaccine development, and changing screening frequency on national Pap test volume: projections from the National Health Interview Survey (NHIS). Cancer 2007;111:34-40.

61 Soni A. Use of the Pap test as a cancer screening tool among women aged 18-64, US noninstitutionalized population, 2005. Statistical brief No 173. Rockville, MD: Medical Expenditure Panel Survey, Agency for Healthcare Research and Quality, 2007.

62 Insinga RP, Glass AG, Rush BB. Pap screening in a US health plan. Cancer Epidemiol Biomarkers Prev 2004;13:355-60.

63 Franco EL, Cuzick J, Hildesheim A, de Sanjose S. Chapter 20: issues in planning cervical cancer screening in the era of HPV vaccination. Vaccine 2006;24(suppl 3):S171-7.

64 Hamilton BE, Martin JA, Ventura SJ. Births: preliminary data for 2006. Natl Vital Stat Rep 2007;55:1-18.

65 Dasbach EJ, Elbasha EH, Insinga RP. Mathematical models for predicting the epidemiologic and economic impact of vaccination against human papillomavirus infection and disease. Epidemiol Rev 2006;28:88-100
66 Newall AT, Beutels P, Wood JG, Edmunds WJ, Macintyre CR. Costeffectiveness analyses of human papillomavirus vaccination. Lancet Infect Dis 2007;7:289-96.

67 Barnabas RV, Kulasingam SL. Economic evaluations of human papillomavirus vaccines. Expert Rev Pharmacoeconomics Outcomes Res 2007;7:1-17.

68 Kim JJ, Brisson M, Edmunds WJ, Goldie SJ. Modeling cervical cancer prevention in developed countries. Vaccine 2008;26(suppl 10):K76-86.

69 Marra F, Cloutier K, Oteng B, Marra C, Ogilvie G. Effectiveness and cost effectiveness of human papillomavirus vaccine: a systematic review. Pharmacoeconomics 2009;27:127-47.

70 Slade BA, Leidel L, Vellozzi C, Woo EJ, Hua W, Sutherland A, et al. Postlicensure safety surveillance for quadrivalent human papillomavirus recombinant vaccine. JAMA 2009;302:750-7.

71 Haug C. The risks and benefits of HPV vaccination. JAMA 2009;302:795-6.

72 Steinbrook R. Health care and the American Recovery and Reinvestment Act. N Engl J Med 2009;360:1057-60.

Accepted: 9 September 2009 